\title{
On Wave Solutions of Gauge-Invariant Generalization of Field Theories with Asymmetric Fundamental Tensor in a Generalized Peres Space-Time $\left(^{*}\right)(* *)$.
}

\author{
Avirudh Pradhan (Gorakhpur, India)
}

Summary. - The gauge invariant generatization of field theories with asymmetric fundamental tensor developed by Buchdahl has been considered and its plane wave-like solutions in the sense of Takeno are investigated in generalized Peres space-time, recently considered by the author. It has been shown that under certain conditions these solutions become identical with those of strong field equations of Einstein in the same space-time. It has been also shown that this space-time satisfying the field equations of Buchdahl admits a parallel null vector field and is gravitationally null which further, transforms to other well known forms of spacetime under a new time coordinate $Z=z-t$.

\section{1. - Introduction.}

Combining the unified field theories of WerL [1] $\left(^{1}\right)$ and Einstein [2], BuchDAHL ([3], [4]) has developed a gauge-invariant theory based on an asymmetric covariant tensor $g_{i j}$ and a covariant vector $K_{i}$ relating these to an asymmetric linear connection $L_{j k}^{i}$ in such a way that this new theory can be regarded equivalently as "gauge-invariant generalization of Einstein's non-symmetric theory" or "the asymmetric generalization of Weyl's theory ".

In Buchdahl's theory [4] the asymmetric tensor $g_{i j}=h_{i j}+f_{i j}$ (where $h_{i j}$ is the symmetric part of $g_{i j}$ which coincides with the fundamental tensor of the metric space and $f_{i j}$ is skew-symmetric parts of $g_{i j}$ ), a covariant vector $K_{i}$ and the linear connection $L_{j k}^{i}$ are defined by

$$
\begin{aligned}
& g_{l i} \Gamma_{i k}^{l}+g_{i l} \Gamma_{k j}^{l}-g_{i j, k}=0, \\
& g_{l j} \gamma_{i k}^{l}+g_{i l} \gamma_{k j}^{l}-g_{i j} K_{k}=0 \\
& L_{j k}^{i}=\Gamma_{j k}^{i}-\gamma_{j k}^{i}
\end{aligned}
$$

(*) Entrata in Redazione il 2 afosto 1976.

(**) Work is supported by State Council of Science and Technology (U.P.), India.

(1) Numbers in brackets refer to the references at the end of the paper. 
where $\Gamma_{j k}^{i}$ is the linear connection of Einstein's non-symmetric theory [2]. The indices $i, j, k, \ldots$ take the values $1,2,3,4$ and a comma $($,$) before an index k$ denotes its partial differentiation with respect to $x^{k}$. The simplest field equations based upon this theory, as given by BUCHDAHL [4], are

$$
\begin{aligned}
& L_{i} \equiv L_{[i j]}^{j}=0,
\end{aligned}
$$

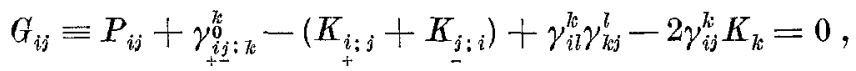

where $P_{i, j}$ is defined by

$$
P_{i j}=-\Gamma_{i j, k}^{k}+\frac{1}{2}\left\{\Gamma_{(i j), j}^{k}+\Gamma_{(k j), i}^{k}\right\}+\Gamma_{i l}^{k} \Gamma_{k j}^{l}-\Gamma_{i j}^{k} \Gamma_{(k j)}^{l}
$$

A semicolon (;) stands for covariant differentiation with respect to $\Gamma_{j k}^{i}$, the sign " + " or "- " or " 0 " below an index fixes the position of covariant index $k$ in connection as $\Gamma_{0}^{0} k, \Gamma_{k 0}^{0}, \Gamma_{(0 k)}^{0}$ and parenthesis ( ) and bracket [ ] including two indices denote the symmetry and antisymmetry respectively between them. Tensors $G_{i j}$ and $P_{i j}$ are the hermitian tensors of gauge invariant and Einstein's usual asymmetric theory respectively.

Further BuchDAHL [4] has considered the field eq. (1.4) and (1.5) in a static spherically symmetric space-time and the possible physical significance of such field equations is briefly discussed. LAL and KHARE [5] have obtained the solutions of these field equations in a $V_{2} \times V_{2}$ space-time. Recently, LAL and SINGH ([6], [7]) have investigated the cylindrical wave solutions of Buchdahl's field equations. Wave solutions of Buchdahl's field equations have been also considered in a generalized Takeno space-time by LaL and SRIVASTAVA [8].

In the present paper we have considered gauge-invariant generalization of Einstein's field equations as given by Buchdahl in a generalized Peres space-time and it has been exhibited that under certain conditions solutions of these field equations exist.

The author [9] has investigated the plane wave-like solutions of field equations of non-symmetric unified field theories of Einstein, Bonnor and Schrödinger by talking the non-sýmmetric fundamental tensor $g_{i j}=h_{i j}+f_{i j}$, given by

$$
g_{i j}=\left[\begin{array}{rrcc}
-A & 0 & \varrho & -\varrho \\
0 & -B & \sigma & -\sigma \\
-\varrho & -\sigma & -(1-E) & -E \\
\varrho & \sigma & -E & 1+E
\end{array}\right]
$$


where $A, B, E, \varrho$ and $\sigma$ are functions of $(x, y, Z), Z=(z-t)$ and

$$
h_{i j}=\left[\begin{array}{rccc}
-1 / A & 0 & 0 & 0 \\
0 & -1 / B & 0 & 0 \\
0 & 0 & -(1+E) & 0 \\
0 & 0 & -E & 1-E
\end{array} \mid .\right.
$$

We use $h_{i j}$ and its conjugate $h^{i j}$ to lower and raise the indices of a tensor.

\section{2. - Solutions of equations (1.1) and (1.2) and evaluation of connections $L_{i j}^{k}$.}

Equation (1.1) is the first equation of Einstein non-symmetric theory and it has been solved in [9].

We shall use Hlavaty [10] method to solve eq. (1.2). Mishra [11] has proved that $\gamma_{i j}^{k}$ can be put into the form

$$
\gamma_{i j}^{k}=H_{i j}^{k}+\beta_{i j}^{k}+U_{i j}^{k},
$$

where

$$
\begin{aligned}
& U_{i j}^{k}=2 h^{k m} S_{m(i j i j) l}^{l}, \\
& H_{i j}^{k}=\frac{1}{2} h^{k l}\left(K_{i} h_{i l}+K_{i} h_{i l}-K_{l} h_{i j}\right), \\
& S_{i j}^{k}=\gamma_{[i j]}^{k}=h^{k l}\left(K_{i j l}^{\prime}+2 U_{b[i j] \mathrm{m}}^{m}\right), \\
& \text { a) } \quad K_{i j k}^{\prime}=K_{i j k}+2 f_{n[i} H_{j] k}^{k}, \\
& \text { b) } \quad K_{i j k}=\frac{1}{2}\left(K_{j} f_{i k}+K_{i} f_{i j}-K_{i} f_{j k}\right) .
\end{aligned}
$$

As $f_{i j}$ given by (1.7) are of the third class in the sense of HLAVATY [10], we have solution of $(2.4)$ as

$$
S_{i j}^{k}=h^{k l}\left(K_{i j l}^{\prime}-2 f_{[i}^{m} K_{i j m n}^{\prime} f_{l}^{n}\right)
$$

Using (1.7) in (2.3) and $(2.5 b)$, we bave the components of $H_{j i}^{k}\left(=H_{i j}^{k}\right)$ and the non-zero components of $K_{i j \cdot k}\left(=-K_{j q k}\right)$ as follows:

$$
\begin{aligned}
& H_{11}^{k}=\left[\frac{1}{2} K_{1}-\frac{1}{2} A K_{2} / B,\left\{-\frac{1}{2} A K_{3}-\frac{1}{2}\left(K_{3}+K_{4}\right) A E\right\},\left\{\frac{1}{2} A K_{4}-\frac{1}{2}\left(K_{3}+K_{4}\right) A E\right\}\right], \\
& H_{22}^{k}=\left[-B K_{1} / 2 A, \frac{1}{2} K_{2},\left\{-\frac{1}{2} B K_{3}-\frac{1}{2}\left(K_{3}+K_{4}\right) B E\right\},\left\{\frac{1}{2} B K_{4}-\frac{1}{2}\left(K_{3}+K_{4}\right) B E\right\}\right],
\end{aligned}
$$


$H_{38}^{k}=\left[-(1-E) K_{1} / 2 A,-(1-E) K_{2} / 2 B,\left\{\frac{1}{2}\left(1+E^{2}\right) K_{3}-\frac{1}{2} E(1-E) K_{4}\right\}\right.$,

$$
\left.\left\{-\frac{1}{2} E(1-E) K_{3}+\frac{1}{2}(1-E)^{2} K_{4}\right\}\right]
$$

$$
H_{44}^{k}=\left[(1+E) K_{1} / 2 A,(1+E) K_{2} / 2 B,\left\{\frac{1}{2}(1+E)^{2} K_{3}+\frac{1}{2} E(1+E) K_{4}\right\},\right.
$$

$$
\left.\left\{\frac{1}{2} E(1+E) K_{3}+\frac{1}{2}\left(1+E^{2}\right) K_{4}\right\}\right] \text {, }
$$

$H_{12}^{k}=\left[\frac{1}{2} K_{2}, \frac{1}{2} K_{1}, 0,0\right]$,

$H_{13}^{k}=\left[\frac{1}{2} K_{3}, 0, \frac{1}{2} K_{1}, 0\right]$,

$H_{14}^{k}=\left[\frac{1}{2} K_{4}, 0,0, \frac{1}{2} K_{1}\right]$,

$H_{23}^{k}=\left[0, \frac{1}{2} K_{3}, \frac{1}{2} K_{2}, 0\right]$,

$H_{24}^{k}=\left[0, \frac{1}{2} K_{4}, 0, \frac{1}{2} K_{2}\right]$,

$H_{34}^{k}=\left[-E K_{1} / 2 A,-E K_{2} / 2 B,\left\{-\frac{1}{2} E(1+E) K_{3}+\frac{1}{2}\left(1-E^{2}\right) K_{4}\right\}\right.$,

$\left.\left\{\frac{1}{2}\left(1-E^{2}\right) K_{3}+\frac{1}{2} E(1-E) K_{4}\right\}\right]$,

and

$$
\begin{aligned}
& K_{123}=-K_{124}=\frac{1}{2}\left(\varrho K_{2}-\sigma K_{1}\right), \quad K_{131}=-K_{141}=\varrho K_{1}, \\
& K_{132}=-K_{142}=K_{231}=-K_{241}=\frac{1}{2}\left(\varrho K_{2}+\sigma K_{1}\right), \\
& K_{133}=\varrho K_{8}, \quad K_{144}=-\varrho K_{4}, \quad K_{134}=K_{143}=-\frac{1}{2} \varrho\left(K_{3}-K_{4}\right), \\
& K_{232}=-K_{242}=\sigma K_{2}, \quad K_{233}=\sigma K_{3}, \quad K_{244}=-\sigma K_{4}, \\
& K_{234}=K_{243}=-\frac{1}{2} \sigma\left(K_{3}-K_{4}\right), \quad K_{341}=-\frac{1}{2} \varrho\left(K_{8}+K_{4}\right), \quad K_{342}=-\frac{1}{2} \sigma\left(K_{3}+K_{4}\right)
\end{aligned}
$$

When use is made of (1.7), (2.7) and (2.8) in equation $(2.5 a)$, we find the values of $K_{i j k}^{\prime}\left(=-K_{j i k}^{\prime}\right)$ as follows:

$$
\begin{aligned}
& K_{131}^{\prime}=-K_{141}^{\prime}=K_{2} \sigma A / 2 B, \quad K_{132}^{\prime}=-K_{142}^{\prime}=-\frac{1}{2} K_{2} \varrho, \\
& K_{133}^{\prime}=-K_{134}^{\prime}=\frac{1}{2}\left\{\varrho K_{4}-\varrho E\left(K_{3}+K_{4}\right)\right\}, \\
& K_{143}^{\prime}=-K_{144}^{\prime}=\frac{1}{2}\left\{\varrho K_{3}+\varrho E\left(K_{3}+K_{4}\right)\right\}, \\
& K_{231}^{\prime}=-K_{241}^{\prime}=-\frac{1}{2} \sigma K_{1}, \quad K_{232}^{\prime}=-K_{242}^{\prime}=K_{2} \varrho B / 2 A, \\
& K_{233}^{\prime}=-K_{234}^{\prime}=\frac{1}{2}\left\{\sigma K_{4}-\sigma E\left(K_{3}+K_{4}\right)\right\}, \\
& K_{243}^{\prime}=-K_{244}^{\prime}=\frac{1}{2}\left\{\sigma K_{3}+\sigma E\left(K_{3}+K_{4}\right)\right\}, \\
& K_{343}^{\prime}=-K_{344}^{\prime}=-\frac{1}{2}\left(\varrho K_{1} / A+\sigma K_{2} / B\right), \\
& K_{121}^{\prime}=-\frac{1}{2} \sigma A\left(K_{3}+K_{4}\right), \quad K_{122}^{\prime}=\frac{1}{2} \varrho B\left(K_{3}+K_{4}\right), \\
& K_{341}^{\prime}=K_{342}^{\prime}=K_{123}^{\prime}=K_{124}^{\prime}=0 .
\end{aligned}
$$


ANIRUdH PradHax: On wave solutions of gauge-invariant generalization, etc. 369

Substituting (1.7) and (2.9) in (2.6) we find that the last term on the right hand side of (2.6) is identically zero and consequently equation (2.6) reduces to

$$
\beta_{i j}^{k}=h^{k l} K_{i j l}^{\prime} .
$$

Using (1.8) and (2.9) in (2.10), the components of $S_{i j}^{k}\left(=-S_{j i}^{k}\right)$ are found to be

$$
\begin{aligned}
& S_{12}^{1}=\frac{1}{2} \sigma\left(K_{3}+K_{4}\right), \quad S_{13}^{1}=-S_{14}^{1}=-\sigma K_{2} / 2 B, \\
& S_{12}^{2}=-\frac{1}{2} \varrho\left(K_{3}+K_{4}\right), \quad S_{13}^{2}=-S_{14}^{2}=\varrho K_{2} / 2 B, \\
& S_{23}^{1}=-S_{24}^{1}=\sigma K_{1} / 2 A, \quad S_{13}^{3}=S_{13}^{4}=-\frac{1}{2} \varrho\left\{K_{4}-E\left(K_{3}+K_{4}\right)\right\}, \\
& S_{23}^{2}=-S_{24}^{2}=-\varrho K_{1} / 2 A, \quad S_{14}^{3}=S_{14}^{4}=-\frac{1}{2} \varrho\left\{K_{3}+E\left(K_{3}+K_{4}\right)\right\}, \\
& S_{23}^{3}=S_{23}^{4}=-\frac{1}{2} \sigma\left\{K_{4}-E\left(K_{3}+K_{4}\right)\right\}, \quad S_{34}^{3}=S_{34}^{4}=\frac{1}{2}\left(\varrho K_{1} / A+\sigma K_{2} / B\right), \\
& S_{24}^{3}=S_{24}^{4}=-\frac{1}{2} \sigma\left\{K_{3}+E\left(K_{3}+K_{4}\right)\right\}, \quad S_{34}^{1}=S_{34}^{2}=S_{12}^{3}=S_{12}^{4}=0 .
\end{aligned}
$$

Equation (2.2) with the help of equations $(1.7),(1.8)$ and $(2.11)$ identically reduces to

$$
U_{i j}^{k}=0 \text {. }
$$

Thus we have solved (2.2), (2.3) and (2.4) in terms of $g_{i j}$ and $K_{i}$. Using equations (2.7), (2.11) and (2.12) we find the components of $\gamma_{i j}^{k}$ as follows:

$$
\begin{aligned}
& \gamma_{11}^{k}=\left[\frac{1}{2} K_{1},-A K_{2} / 2 B,-\frac{1}{2} A\left\{K_{3}+E\left(K_{3}+K_{4}\right)\right\}, \frac{1}{2}\left\{K_{4}-E\left(K_{3}+K_{4}\right)\right\}\right], \\
& \gamma_{22}^{k}=\left[-B K_{1} / 2 A, \frac{1}{2} K_{2},-\frac{1}{2} B\left\{K_{3}+E\left(K_{3}+K_{4}\right)\right\}, \frac{1}{2}\left\{K_{4}-E\left(K_{3}+K_{4}\right)\right\}\right] \text {, } \\
& \gamma_{33}^{k}=\left[-K_{1}(1-E) / 2 A,-K_{2}(1-E) / 2 B, \frac{1}{2}\left\{K_{3}-E K_{4}+E^{2}\left(K_{8}+K_{4}\right)\right\}\right. \text {, } \\
& \left.\frac{1}{2}\left\{K_{4}-E K_{3}-2 E K_{4}+E^{2}\left(K_{3}+K_{4}\right)\right\}\right] \text {, } \\
& \gamma_{44}^{k}=\left[K_{1}(1+E) / 2 A, K_{2}(1+E) / 2 B, \frac{1}{2}\left\{K_{3}+2 E K_{3}+E K_{4}+E^{2}\left(K_{3}+K_{4}\right)\right\}\right. \text {, } \\
& \left.\frac{1}{2}\left\{K_{4}+E K_{3}+E^{2}\left(K_{3}+K_{4}\right)\right\}\right], \\
& \gamma_{\frac{12}{2 i}}^{k_{2}}=\left[\frac{1}{2} K_{2} \pm \frac{1}{2} \sigma\left(K_{3}+K_{4}\right), \frac{1}{2} K_{1} \mp \frac{1}{2} \varrho\left(K_{3}+K_{4}\right), 0,0\right] \text {, } \\
& \gamma_{31}^{k}=\left[\frac{1}{2} K_{3} \mp \frac{1}{2} \sigma K_{2} / B, \pm \varrho K_{2} / 2 B, \frac{1}{2} K_{1} \mp \frac{1}{2} \varrho\left\{K_{4}-E\left(K_{3}+K_{4}\right)\right\}\right. \text {, } \\
& \left.\mp \frac{1}{2} \varrho\left\{K_{4}-E\left(K_{3}+K_{4}\right)\right\}\right] \text {, } \\
& \gamma_{41}^{k}=\left[\frac{1}{2} K_{4} \pm \sigma K_{2} / 2 B, \mp \varrho K_{2} / 2 B, \mp \frac{1}{2} \varrho\left\{K_{3}+E\left(K_{3}+K_{4}\right)\right\}\right. \text {, } \\
& \left.\frac{1}{2} K_{1} \mp \frac{1}{2} \varrho\left\{K_{3}+E\left(K_{3}+K_{4}\right)\right\}\right] \text {, } \\
& \gamma_{\frac{23}{32}}^{k}=\left[ \pm \sigma K_{1} / 2 A, \frac{1}{2} K_{3} \mp \varrho K_{1} / 2 A, \frac{1}{2} K_{2} \mp \frac{1}{2} \sigma\left\{K_{4}-E\left(K_{3}+K_{4}\right)\right\}\right. \text {, } \\
& \left.\mp \frac{1}{2} \sigma\left\{K_{4}-E\left(K_{3}+K_{4}\right)\right\}\right] \text {, } \\
& \gamma_{24}^{k}=\left[\mp \sigma K_{1} / 2 A, \frac{1}{2} K_{4} \pm \varrho K_{1} / 2 A, \mp \frac{1}{2} \sigma\left\{K_{3}+E\left(K_{3}+K_{4}\right)\right\}\right. \text {, } \\
& \left.\frac{1}{2} K_{2} \mp \frac{1}{2} \sigma\left\{K_{3}+E\left(K_{3}+K_{4}\right)\right\}\right] \text {, } \\
& \gamma_{34}^{k}=\left[-E K_{1} / 2 A,-E K_{2} / 2 B, \frac{1}{2}\left\{-E(1+E) K_{3}+\left(1-E^{2}\right) K_{4} \pm\right.\right. \\
& \left. \pm\left(\varrho K_{1} / A+\sigma K_{2} / B\right\}, \frac{1}{2}\left\{\left(1-E^{2}\right) K_{3}+E(1-E) K_{4} \pm\left(\varrho K_{1} / A+\sigma K_{2} / B\right)\right\}\right] .
\end{aligned}
$$


ANIRUDH PRADHAN: On wave solutions of gauge-invariant generalization, etc.

Substituting the values of $\Gamma_{i j}^{k}$ from [9] and $\gamma_{i j}^{k}$ from $(2,13)$ into (1.3) we get the components of $L_{i j}^{k}$.

\section{3. - Solution of equation (1.4).}

Using the components of $\Gamma_{i j}^{k}$ from [9] and $\gamma_{i j}^{k}$ from (2.13) in equation (1.4) we find that when $i=1$ and 2 equations (1.4) give

$$
K_{3}+K_{4}=0
$$

while when $i=3$ and 4 equations (1.4) give

$$
2\left(\varrho K_{1} B+\sigma K_{2} A\right)-2\left(B \varrho_{x}+A \sigma_{y}\right)+B \varrho\{\log (A / B)\}_{\infty}+A \sigma\{\log (B / A)\}_{y}=0
$$

From hereonwards, the lower suffixes $x$ and $y$ attached with any function indicate partial differentiation with respect to $x$ and $y$ respectively while an overhead bar stands for partial differentiation with respect to $Z$.

Thus (3.1) and (3.2) are necessary conditions in order that the gauge-invariant field equation (1.4) be satisfied.

4. - Tensors $G_{i j}$ and $P_{i j}$.

The gauge-invariant Einstein tensor $G_{i j}$, as given by BuchdaHL [4], is

$$
\begin{gathered}
G_{i j}=P_{i j}-\left(K_{i, j}+K_{j, i}-2 K_{1} \Gamma_{i j}^{l}\right)-2 \gamma_{i j}^{l} K_{1}+\gamma_{i j, l}^{l}+ \\
\Gamma_{(m l)}^{l} \gamma_{i j}^{m}-\Gamma_{i l}^{m} \gamma_{m j}^{l}-\Gamma_{l j}^{m} \gamma_{i m}^{l}+\gamma_{i l}^{m} \gamma_{m j}^{l} .
\end{gathered}
$$

Using the components of $\Gamma_{i j}^{k}$ from [9] we can compute from (1.6) the components of $P_{i j}$ as follows:

$$
\begin{aligned}
P_{11}= & L / B, \quad P_{12}=P_{21}=0, \quad P_{22}=L / A, \\
P_{13}= & -P_{14}=-N / B-H_{x}-(H-I) B_{x} / 2 B+b_{y} / 2 B+\left(A_{y} / A-B_{y} / B\right) b / 4 B, \\
P_{31}= & -P_{41}=-N / B+H_{x}+(H-I) B_{x} / 2 B-b_{y} / 2 B-\left(A_{y} / A-B_{y} / B\right) b / 4 B, \\
P_{23}=- & P_{24}=M / A-I_{y}+(H-I) A_{y} / 2 A+b_{x} / 2 A-\left(A_{x} / A-B_{x} / B\right) b / 4 A, \\
P_{32}=- & P_{42}=M / A+I_{y}-(H-I) A_{y} / 2 A-b_{x} / 2 A+\left(A_{x} / A-B_{x} / B\right) b / 4 A, \\
P_{33}=- & P_{34}=-P_{43}=P_{44}=\left(\bar{A}-\bar{A}^{2} / 2 A\right) / 2 A+\left(\bar{B}-\bar{B}^{2} / 2 B\right) / 2 B- \\
& S_{x}-T_{y}-H^{2}-I^{2}+\bar{H}+\bar{I}-b^{2} / 2 A B-\frac{1}{2} S\left(A_{x} / A+B_{x} / B\right)- \\
& \frac{1}{2} T\left(A_{y} / A+B_{y} / B\right),
\end{aligned}
$$


where

$$
\begin{aligned}
& 2 L=A_{y y}+B_{x x}-\left(A_{y}^{2}+A_{x} B_{x}\right) / 2 A-\left(B_{x}^{2}+A_{y} B_{y}\right) / 2 B, \\
& 2 M=\bar{A}_{y}-\frac{1}{2} A_{y}(\bar{A} / A+\bar{B} / B), \\
& 2 N=-\bar{B}_{x}+\frac{1}{2} B_{x}(\bar{A} / A+\bar{B} / B), \\
& H=\left(-\varrho_{x}+\varrho A_{x} / 2 A-\sigma A_{y} / 2 B\right) / A, \\
& I=\left(-\sigma_{y}+\sigma B_{y} / 2 B-\varrho B_{x} / 2 A\right) / B, \\
& b=\sigma_{x}+\varrho_{y}-\varrho A_{y} / A-\sigma B_{x} / B, \\
& S=\left\{\frac{1}{2} E_{x}+2 \varrho\left(-\varrho_{x}+\varrho A_{x} / 2 A\right) / A-\sigma\left(b+\varrho A_{y} / A\right) / B\right\} / A, \\
& T=\left\{\frac{1}{2} E_{y}+2 \sigma\left(-\sigma_{y}+\sigma B_{y} / 2 B\right) / B-\varrho\left(b+\sigma B_{x} / B\right) / A\right\} / B .
\end{aligned}
$$

Again using $\Gamma_{i j}^{k}$ from [9], (2.13) and (3.1) in (4.1) the components of $G_{i j}$ are given by

$$
\begin{aligned}
G_{11}= & P_{11}-\frac{3}{2}\left(K_{1}\right)_{, 1}-\frac{1}{2}\left(A K_{2} / B\right)_{, 2}-\frac{1}{2} A\left(K_{3,3}-K_{4,4}\right)+ \\
& 3 K_{1}\left(A_{x} / 4 A\right)-K_{1}\left(B_{x} / 4 B\right)+A K_{2}^{2} / 2 B-A K_{2}\left(A_{y} / A+B_{y} / B\right) / 4 B, \\
G_{22}= & P_{22}-\frac{3}{2}\left(K_{2}\right)_{, 2}-\frac{1}{2}\left(B K_{1} / A\right)_{, 1}-\frac{1}{2} B\left(K_{3,3}-K_{4,4}\right)+ \\
& 3 K_{2}\left(B_{y} / 4 B\right)-K_{2}\left(A_{y} / 4 A\right)+B\left(K_{1}^{2} / 2 A\right)-B K_{1}\left(A_{x} / A+B_{x} / B\right) / 4 A, \\
G_{12}= & G_{21}=K_{1}(A, / 2 A)+K_{2}\left(B_{x} / 2 B\right)-\frac{1}{2} K_{1} K_{2}-\frac{1}{2}\left(K_{1,2}+K_{2,1}\right), \\
G_{13}= & P_{13}-\frac{1}{2}\left(K_{1,3}+K_{3,1}\right)-\frac{1}{2} K_{1} K_{3}-3 b\left(K_{2} / 4 B\right)-\varrho K_{1}^{2} / 2 A- \\
& \varrho K_{2}^{2} / 2 B-\frac{1}{2}\left(\sigma K_{2} / B\right)_{, 1}+\frac{1}{2}\left(\varrho K_{2} / B\right)_{, 2}-\frac{1}{2}\left(\varrho K_{4}\right)_{, 3}- \\
& \frac{1}{2}\left(\varrho K_{4}\right)_{, 4}+K_{1}\left(A / 2 A+H-\frac{1}{2} I\right)+K_{1}\left(\sigma A_{y}+\varrho B_{x}\right) / 4 A B+ \\
& K_{2}\left(\varrho B_{y}-\sigma B_{x}\right) / 4 B^{2}+\xi K_{2} / 2 B \\
G_{31}= & P_{31}-\frac{1}{2}\left(K_{1,3}+K_{3,1}\right)-\frac{1}{2} K_{1} K_{3}+3 b\left(K_{2} / 4 B\right)+\varrho K_{1}^{2} / 2 A+ \\
& \varrho K_{2}^{2} / 2 B+\frac{1}{2}\left(\sigma K_{2} / B\right)_{, 1}-\frac{1}{2}\left(\varrho K_{2} / B\right)_{, 2}+\frac{1}{2}\left(\varrho K_{4}\right)_{, 3}+\frac{1}{2}\left(\varrho K_{4}\right)_{, 4}+ \\
& K_{1}\left(\bar{A} / 2 A-H+\frac{1}{2} I\right)-K_{1}\left(\sigma A_{y}+\varrho B_{x}\right) / 4 A B- \\
& K_{2}\left(\varrho B_{y}-\sigma B_{x}\right) / 4 B^{2}-\xi K_{2} / 2 B, \\
. & . .
\end{aligned}
$$

and the expressions for $G_{14}, G_{41}, G_{23}, G_{32}, G_{24}, G_{42}, G_{33}, G_{34}, G_{44}$ are omitted for brevity's 
sake. Here a comma followed by an index $i$ after a small and curly bracket denotes partial differentiation with respect to $x^{i}$.

There are various possibilities under which the solution of (1.5) may be considered. However, in this paper we consider the case when $K_{1}=K_{2}=0$ and $K_{3}=-K_{4}=\psi(z-t)$.

\section{5. - Solution of equation (1.5).}

If $K_{1}=K_{2}=0$ and $K_{3}=-K_{4}=\psi(z-t)$, then the field equation (1.5) with the help of $(3.2)$ gives

$$
\begin{array}{ll}
\text { a) } & L=0, \\
\text { b) } & N / B+H_{x}+H B_{x} / B-b_{y} / 2 B-\left(A_{y} / A-B_{y} / B\right) b / 4 B=0, \\
\text { c) } & N / B-H_{x}-H B_{x} / B+b_{y} / 2 B+\left(A_{y} / A-B_{y} / B\right) b / 4 B=0, \\
\text { d) } \quad & M / A+H_{y}+H A_{y} / A+b_{x} / 2 A-\left(A_{x} / A-B_{x} / B\right) b / 4 A=0, \\
\text { e) } \quad M / A-H_{y}-H A_{y} / A-b_{x} / 2 A+\left(A_{x} / A-B_{x} / B\right) b / 4 A=0, \\
\text { f) } \quad\left(\bar{A}-\bar{A}^{2} / 2 A\right) / 2 A+\left(\bar{B}-\bar{B}^{2} / 2 B\right) / 2 B-B_{x}-T_{y}-2 H^{2}-b^{2} / 2 A B- \\
& \frac{1}{2} S\left(A_{x} / A+B_{x} / B\right)-\frac{1}{2} T\left(A_{y} / A+B_{y} / B\right)-\bar{\psi}-\frac{1}{2} \psi^{2}-0 .
\end{array}
$$

From equations $(5.1 b)$ and $(5.1 c)$, we get

$$
\text { a) } N=0 \text { and b) } 2(B H)_{x}-b_{y}-\frac{1}{2} b\{\log (A / B)\}_{y}=0 \text {. }
$$

From equations $(5.1 d)$ and (5.1e), we have

$$
\text { a) } M=0 \text { and } \quad \text { b) } 2(A H)_{y}+b_{x}-\frac{1}{2} b\{\log (A / B)\}_{x}=0 \text {. }
$$

Equation (5.1a) ean be written as

$$
\left(B_{x} / \sqrt{A \bar{B}}\right)_{x}+\left(A_{y} / \sqrt{A B}\right)_{y}=0 .
$$

Equations (5.2a) and (5.3a), after integration, reduce to

$$
\text { a) } B_{x} / \sqrt{A B}=C_{1} \quad \text { and } \quad \text { b) } A_{y} / \sqrt{A \bar{B}}=C_{2} \text {, }
$$

where $C_{1}$ and $C_{2}$ are some functions of $(x, y)$. Now we have to find the solutions of equations $(5.1 f),(5.2 b),(5.3 b),(5.4)$ and $(5.5)$ only. These equations are non-linear partial differential equations and their solutions are complicated. Hence let us assume

$$
A=B f, \quad(f=f(Z)) .
$$


From equations (0.5) we get

$$
B_{x} A_{y}=A B C_{1} O_{2}
$$

With the help of (5.6), equation (5.7) reduces to

$$
p q=\theta, \quad\left(p=B_{x}, q=B_{y}, \theta=K_{1} K_{2} B^{2}\right),
$$

which is a standard form of differential equation which can be solved by using the standard technique of CHARPIT [12].

By virtue of $(5.6)$, equations $(5.1 f),(5.2 b),(5.3 b)$ and $(5.4)$ reduce to

a) $2 \bar{B}-(\bar{B} / B)^{2}+\bar{B} \bar{f}+\bar{f} / f-\frac{1}{2}(\bar{f} / f)-2 S^{\prime} B_{x} / B-2 T^{\prime} B_{y} / B-$

$$
\left(B_{x}^{\prime}+T_{y}^{\prime}+2 H^{\prime 2}\right)-b^{\prime 2} / B^{2} f-\ddot{\psi}-\frac{1}{2} \psi^{2}=0,
$$

$$
\begin{aligned}
& \text { b) } 2\left(B H^{\prime}\right)_{x}-b_{y}^{\prime}=0, \\
& \text { c) } 2 f\left(B H^{\prime}\right)_{y}+b_{x}^{\prime}=0, \\
& \text { d) } \quad(\log B)_{x x}+f(\log B)_{y y}=0,
\end{aligned}
$$

where $S^{\prime}, T^{\prime}, H^{\prime}$ and $b^{\prime}$ are the values of $S, T, H$ and $b$ when $A=B f$ is used in expressions of $S, T, H$ and $b$ respectively. Hence we have:

The $g_{i j}$ given by (1.7) with (5.6) is a solution of Buchdahl's field equations under condition (5.9).

Theorkm 5.1. - If $K_{1}=K_{2}=0$ and $K_{3}=K_{4}=\psi(z-t)$ then the necessary and sufficient condition that the solution of gauge-invariant generalized field equations of Einstein be a solution of strong field equations of Einstein's unified field theory in the generalized Peres space-time is that $\psi$ must be of the form

$$
\psi=\left(C_{3}+\frac{1}{2} Z\right)^{-1}, \quad O_{3} \text { is some function of }(x, y) \text {. }
$$

Proof. - We have shown that if $K_{1}=K_{2}=0$ and $K_{3}=-K_{4}=\psi(z-t)$ then gange-invariant generalized field equation of Einstein, i.e. $G_{i j}=0$ reduces to (5.1).

If the solution of gauge-invariant generalized field equations of Einstein are those of strong field equations of Einstein's unified field theory then $Q_{i j}=P_{i j}=0$ which by virtue of equation (5.1) gives $\bar{\psi}+\frac{1}{2} \psi^{2}=0$ which on integration gives $\psi=\left(C_{3}+\frac{1}{2} Z\right)^{-1}$.

Conversely if (5.10) holds then by differentiating it we get $\ddot{\psi}+\frac{1}{2} \psi^{2}=0$, which reduces (5.1) to $G_{i j}=0$, i.e. $G_{i j}=P_{i j}=0$. Hence the condition is sufficient. 


\section{6. - Special case.}

If we set $C_{1}=C_{2}=0$ then from equations (5.5) we find that $B_{x}=A_{y}=0$ and in this case the $g_{i j}$ given by (1.7) where $A=A(x, Z), B=B(y, Z)$ and $E=E(x, y, Z)$ constitute the wave solutions of Buchdah's field equations provided the following relations hold:

a) $2\left(B H^{*}\right)_{x}-\sigma_{x y}-\varrho_{y y}+\left(\sigma_{x}+\varrho_{y}\right) B_{y} / 2 B=0$,

b) $2\left(A H^{*}\right)_{x}-\sigma_{x x}-\varrho_{x y}-\left(\sigma_{x}+\varrho_{y}\right) A_{x} / 2 A=0$,

c) $\left(\bar{A}-\bar{A}^{2} / 2 A\right) / A-\left(\bar{B}-\bar{B}^{2} / 2 B\right) / B-S^{*} A_{x} / A-T^{*} B_{y} / B-$

$$
2\left(S_{x}^{*}+T_{y}^{*}+2 H^{* 2}\right)-\left(\sigma_{x}+\varrho_{y}\right)^{2}-\bar{\psi}-\frac{1}{2} \psi^{2}=0,
$$

where $S^{*}, T^{*}$ and $H^{*}$ are the values of $S, T$, and $H$ respectively when $A=A(x, Z)$ and $B=B(y, Z)$.

REMARK. - Here we add some words concerning the solution obtained in Section 6. When $C_{1}=C_{2}=0$ then the space-time $h_{i j}$ representing the gravitational potential takes the form

$$
\begin{aligned}
& d s^{2}=-A d x^{2}-B d y^{2}-(1-E) d z^{2}-2 E d z d t+(1+E) d t^{2} \\
& A=A(x, Z), \quad B=B(y, Z), \quad E=E(x, y, Z) .
\end{aligned}
$$

The space-time defined by (6.2) being gravitationally null admits a parallel null vector field $v^{i}=(0,0,1,1)$ (or $c v^{i}, c$ being constant). Here it should be mentioned that the space-time represented by the metric (6.2) is a generalization of Peres spacetime [13], and it also becomes $H$ space-time studied by TAkEno [14], Bondi-PrRaniRobinson [15] and SHIbata [16] when we assume $=A(Z), B=B(Z)$ and $E=E(Z)$.

The solutions found out in this paper can be called plane wave-like solutions following TAKENO [14] and the wave is propagating in the positive direction of $z$-axis with unit velocity.

Acknowledgment. Thanks are due to Prof. K. B. LAL and Dr. T. SingH for helpful discussions.

\section{REFERENCES}

[1] H. WeYL, Raum, Zeit, Materie, 3rd ed., Berlin (1919).

[2] A. Ensstern, The meaning of relativity, 5th ed. revised, London (1955), Appendix II.

[3] H. A. BUCHDAHL, Gauge invariant generalization of field theories with asymmetric fun. damental tensor, Quart. J. Math., Oxford, 8 (1957), pp. 89-96. 
[4] H. A. BUCHDAHL, Gauge invariant generalization of field theories with asymmetric fundamental tensor II, Quart. J. Math., Oxford, 9 (1958), pp. 257-264.

[5] K. B. LAI - D. C. KHARE, On wave solutions of gauge invariant generalization of field theories with asymmetric fundamental tensor in $\nabla_{2} \times V_{2}$ space-time, Tensor, N.S., 21 (1970), pp. 333-339.

[6] K. B. LAL - T. SINGH, On cylindrical wave solutions of gauge invariant generalization equations of field theories with asymmetrio fundamental tensor, Tensor, N. S., 23 (1972), pp. $185-192$.

[7] K. B. LAL - T. SINGH, On cylindrical wave solutions of gauge invariant generalization equations of field theories with asymmetric fundamental tensor (II), Tensor, N.S., 24 (1972), pp. 217-223.

[8] K. B. LAL - R. P. SRIVAStaVa, On the wave solutions of gauge invariant generalization of field theories with asymmetric fundamental tensor in a generalized Takeno space-time, Tensor, N. S., 27 (1973), pp. 7-15.

[9] A. Pradhan, On wave solutions of the non-symmetric unified field theories, Rov. Mat. $Y$ Fis. Teorica, 27 (1977), in press.

[10] V. HLaVaty, Geometry of Einstein's unified field theory, Noordhoff, Groningen (1957).

[11] R. S. MishrA, Solutions of gauge invariant generalization equations of the field theories with asymmetric fundamental tensor, Quart. J. Math., Oxford, 14 (1963), pp. 81-85.

[12] I. N. SNEDdon, Elements of Partial Differential Equations, McGraw-Hill Kogakusha, Ltd. (1957), Chap. II.

[13] A. Perks, Some gravitational waves, Phys. Rev. Lett., 3 (1959), pp. 571-572.

[14] H. TAKeNo, The mathematical theory of plane gravitational waves in general relativity, Sci. Rep. Res. Inst. Theor. Phys. Hir. Univ., I (1961).

[15] H. Bondi - F. A. Pirani - I. Robinson, Gravitational waves in general relativity. . III : Exact plane waves, Proc. Roy. Soc., London, Ser. A, 251 (1959), p. 519.

[16] T. Shibata, Geometrical definition of plane gravitational waves in general relativity, Mem. Fac. Eng., Hiroshima University, 1 (1960), pp. 165-184. 